\title{
Screening of conditions controlling spectrophotometric sequential injection analysis
}

\author{
Abubakr M Idris
}

\begin{abstract}
Background: Despite its potential benefits over univariate, chemometrics is rarely utilized for optimizing sequential injection analysis (SIA) methods. Specifically, in previous vis-spectrophotometric SIA methods, chemometrically optimized conditions were confined within flow rate and reagent concentrations while other conditions were ignored.

Results: The current manuscript reports, for the first time, a comprehensive screening of conditions controlling visspectrophotometric SIA. A new diclofenac assay method was adopted. The method was based on oxidizing diclofenac by permanganate (a major reagent) with sulfuric acid (a minor reagent). The reaction produced a spectrophotometrically detectable diclofenac form. The $2^{6}$ full-factorial design was utilized to study the effect of volumes of reagents and sample, in addition to flow rate and concentrations of reagents. The main effects and all interaction order effects on method performance, i.e. namely sensitivity, rapidity and reagent consumption, were determined. The method was validated and applied to pharmaceutical formulations (tablets, injection and gel).

Conclusions: Despite 64 experiments those conducted in the current study were cumbersome, the results obtained would reduce effort and time when developing similar SIA methods in the future. It is recommended to critically optimize effective and interacting conditions using other such optimization tools as fractional-factorial design, response surface and simplex, rather than full-factorial design that used at an initial optimization stage. In vis-spectrophotometric SIA methods those involve developing reactions with two reagents (major and minor), conditions affecting method performance are in the following order: sample volume $>$ flow rate $\approx$ major reagent concentration $>>$ major reagent volume $\approx$ minor reagent concentration $>>$ minor reagent volume.
\end{abstract}

\section{Background}

Sequential injection analysis (SIA) is the second generation of an extended family called flow injection (FI) techniques [1]. SIA gathers valuable advantages, including automation, miniaturization, versatility and costeffectiveness, over other generations and versions of FI techniques. Recent articles reviewing the principles, developments and applications of FI techniques are available elsewhere $[2,3]$.

On the other hand, optimizing experimental conditions is a prior in developing analytical methods. A literature survey was carried out by the Scopus ${ }^{\mathbb{B}}$ database using the phrase "sequential injection analysis". Since the introduction of SIA technique in 1990 [1], the survey has enumerated 639 articles. Within the extracted

Correspondence: abubakridris@hotmail.com

Department of Chemistry, College of Science, King Faisal University, P.O. Box 400, Hofuf 31982, Saudi Arabia results, a further literature survey, using the keywords "chemometrics" or "multivariate", was carried out. In the latter survey, thirty-nine articles were found, i.e. the rest of articles reported developing SIA methods using the univariate approach.

The univariate approach optimizes conditions one-byone by varying levels of one condition while levels of other conditions are held at constant levels. This procedure makes the univariate approach time- and reagentconsuming. Moreover, the univariate approach is unable to consider interaction effect between conditions and hence the maximum efficiency of analytical methods might not be obtained.

On the other hand, chemometrices, as a group of multivariate approaches, is more powerful than the univariate approach. The strategy of chemometrics is that to obtain the highest efficiency of analytical methods in the shortest way. Hence, chemometrices reduces consumption of 
reagents and sample, besides it saves time and minimizes effort. Chemometrics gains its strategy throughout the following ways: (i) examining the effect of conditions and their interactions on the efficiency of analytical methods, (ii) optimizing conditions with considering their interactions, (iii) developing more than one analytical aspect at the same time, (iv) reducing a large amount of data that can be easily interpreted and (v) testing the ruggedness [4-6].

Among the most common effective chemometric optimization approaches are the experimental design-based methods. The remarkable applications of experimental design include factor screening, response surface examination, system optimization and system robustness. Factorial design, which is the dominant factor screening method, allows to select which factors are significant and at what levels [4-6].

On the other side, for its selectivity, simplicity and familiarity, spectrophotometric detection is frequently used with SIA [7-10]. In SIA with UV detection, multivariate curve resolution with alternating least squares (MCR-ALS), as a chemometric tool, was successfully utilized to treat second-order data in order to optimize resolution using UV detection [11-15]. However, the most applied spectrophotometric detection that used with SIA methods is in the vis range, which is more selective. In those methods, chromogenic reactions, which most probably are redox, complexation, ion pairing and charge transfer, are usually applied. Most of those reactions involved two reagents or more [16-26]. In those methods, the chemometrically optimized conditions limited within flow rate and concentrations of reagents while other such effective conditions as volumes of reagents and sample were neglected.

Therefore, it has been proposed, for the first time, to screen conditions controlling vis-spectrophotometric SIA methodologies. An issue that would reduce effort and time when developing new methods in the future. As an example, a new vis-spectrophotometric SIA method for the assay of diclofenac was adopted.

Diclofenac is chemically named 2-[(2,6-dichlorophenyl)aminophenyl]-acetic acid (Figure 1). It is a potent analgesic and anti-inflammatory agent. Due to its use

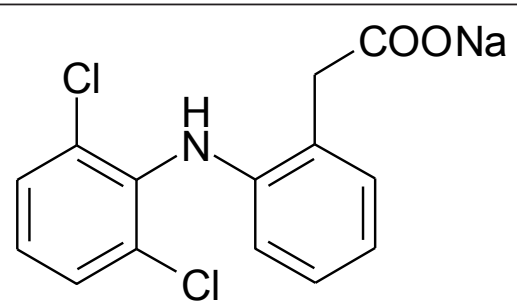

Figure 1 Chemical structure of sodium diclofenac for many treatments, diclofenac is prepared in a wide range of formulations including tablets, capsules, drops, injections, suppositories, gels and ointments. The extensive worldwide use of diclofenac has aroused researchers to develop many assay methods using various analytical techniques. In this issue, it has been found that, within the last five years, more than fifteen methods were reported. Gravimetry [27], spectrophotometry [28,29], fluorometry [30], Raman Spectroscopy [31,32], diffuse reflectance photometry [33], potentiometry [34,35], multisyringe flow injection analysis with amperometry [36], liquid chromatography [37,38], high performance liquid chromatography [39-41], thin layer chromatography [42], high performance thin chromatography [43] and gas chromatography-mass spectrometry [44] were utilized.

\section{Results and Discussion}

\section{Preliminary study}

Recently, permanganate, as the superior oxidizing agent with its high absorptivity, has been found selective in controlled conditions for the assay of some medicines in their formulations $[17,19,26,45,46]$. In the current work, it has been found that diclofenac can be oxidized by permanganate in sulphuric acid media. The oxidized form of diclofenac is spectrophotometrically detectable at $450 \mathrm{~nm}$.

Before undertaking any screening study, it is important to delineate clearly the boundaries of conditions controlling SIA. The minimum and maximum applied levels of conditions are introduced in Table 1.

Regarding levels of flow rate for spectrophotometric measurement, following the practice of SIA $[17-19,21,23,24,26,45,46], 15-30 \mu \mathrm{L} / \mathrm{s}$ is the most suitable range. Flow rate lower than $15 \mu \mathrm{L} / \mathrm{s}$ decreases sample frequency while flow rate higher than $30 \mu \mathrm{L} / \mathrm{s}$ decreases repeatability.

The range of $1.0-5.0 \mathrm{mmol} / \mathrm{L}$ was adopted for permanganate concentration. Higher diclofenac concentration might not be completely oxidized by permanganate concentration lower than the adopted range. On the other hand, at permanganate concentration above the

Table 1 Levels of experimental condition applied for the $2^{6}$ full-factorial design optimization

\begin{tabular}{lcc}
\hline Experimental condition & Minimum level & Maximum level \\
\hline Flow rate $(\mu \mathrm{L} / \mathrm{s})$ & 15 & 30 \\
$\begin{array}{l}\text { Permanganate concentration } \\
(\mathrm{mmol} / \mathrm{L})\end{array}$ & 1.0 & 5.0 \\
Sulphuric acid concentration & 10 & 100 \\
$(\mathrm{mmol} / \mathrm{L})$ & & 100 \\
Permanganate volume $(\mu \mathrm{L})$ & 50 & 60 \\
Sulphuric acid volume $(\mu \mathrm{L})$ & 30 & 60 \\
Diclofenac volume $(\mu \mathrm{L})$ & 30 & \\
\hline
\end{tabular}


adopted range, significant absorbance of diclofenac was not obtained.

For sulfuric acid concentration, a level higher than $100 \mathrm{mmol} / \mathrm{L}$ distorted the base line of a SIA-gram and produced poor repeatability as in previous procedures $[17-19,21,23,24,26,45,46]$. On the contrary, acid concentrations bellow $10 \mathrm{mmol} / \mathrm{L}$ did not record significant absorbance.

The volume ranges of reagents and sample were adopted based upon the criteria of that to obtain significant absorbance and acceptable repeatability. It has been found that, generally, high volume produced non-repeatable results while low volumes decreased absorbance.

\section{Screening of conditions using factorial design}

Unless otherwise described the term "response" refers here to the absorbance of an oxidized form of diclofenac. As mentioned before, the $2^{6}$ full-factorial design was adopted. The base 2 stands for the minimum and the maximum levels of experimental conditions. The power 6 is the number of experimental conditions those would be optimized, which include flow rate, permanganate concentration, sulfuric acid concentration, permanganate volume, sulfuric acid volume and sample volume. A total of 64 experiments using $100 \mu \mathrm{g} / \mathrm{mL}$ diclofenac, as the result of the $2^{6}$ full-factorial design, were conducted. For validation purpose, each experiment was repeated three times, which is practicable when using such a fully-automated technique as SIA.

It was found that the experiment that included the conditions of low flow rate $(15 \mu \mathrm{L} / \mathrm{s})$, high permanganate concentration $(5.0 \mathrm{mmol} / \mathrm{L})$, high acid concentration $(100 \mathrm{mmol} / \mathrm{L})$, low permanganate volume $(50 \mu \mathrm{L})$, high acid volume $(60 \mu \mathrm{L})$ and high sample volume $(60 \mu \mathrm{L})$ recorded the highest response, which was 1.87 . Another experiment also recorded almost the same response, namely 1.77 . The conditions of the latter experiment are the same of the frontal experiment with the exception of the use of low acid volume $(30 \mu \mathrm{L})$.

The main effects " $E$ ", $n=6$, and all interaction order effects, $n=57$, on absorbance were calculated using equation 1 [4-6]. " $y(+1)$ " and " $y(-1)$ " are the absorbance values at the minimum and the maximum levels of an examined factor, respectively. " $n$ " is the number of experiments at one level. " $n$ " in the current design $=32$.

$$
E=\frac{\sum y(+1)}{n}-\frac{\sum y(-1)}{n}
$$

A wide range of grades, ranging from 0.005 to 0.450 , was obtained. To simplify that range, effect factors $>0.1$ were considered significant. It has been found that for effect factor of $<0.1$ the difference in responses at minimum and maximum levels of a condition, e.g. acid volume, with other fixed conditions was almost less than 0.1. From the viewpoint of spectrophotometry, the difference in absorbance of $<0.1$ is insignificant. Figure 2 shows factors of $>0.1$, which is considered, as relatively effective factors. It has been found that the most effective factor is sample volume that positively effect on response. High diclofenac volume increases the number of moles of diclofenac and hence increases absorbance. In the second order of effect is the positive effect of permanganate concentration and negative effect of flow rate (Figure 2). Negative effect of the latter condition indicates slow oxidation reaction of diclofenac. Increasing permanganate concentration with increasing response emphasizes that the oxidation of diclofenac by acidified permanganate is slow. Regarding other main factors, permanganate volume and acid concentration recorded relatively lower effect than other main factors while acid volume did not record significant effect. On the other side, although the effect of both permanganate concentration and flow rate are in the same order, the most significant interaction effect was recorded for sample volume with permanganate concentration.

In order to set up the optimum conditions, it has to compromise between results obtained from factorial design and those obtained from the calculation of effect factors. Primarily, there was no significant difference between the responses obtained from experiments those recorded the responses of 1.87 and 1.77, which differed in acid volume. The results obtained from the calculations of the main and interaction effect factors show that acid volume has the lowest effect (Figure 2). Therefore, the maximum efficiency, in terms of sensitivity, rapidity and reagent consumption, of the proposed SIA method can be extracted from conditions of experiment that recorded the response of 1.77. Consequently, the optimum adopted conditions were minimum flow rate $(15 \mu \mathrm{L} / \mathrm{s})$, maximum permanganate concentration $(5.0 \mathrm{mmol} / \mathrm{L})$, minimum acid concentration $(10 \mathrm{mmol} / \mathrm{L})$, minimum permanganate volume $(50 \mu \mathrm{L})$, minimum acid volume $(30 \mu \mathrm{L})$ and maximum diclofenac volume $(60 \mu \mathrm{L})$.

\section{Method validation}

To examine the linear range and the weighed regression of calibration equation, a long series of diclofenac standard solutions were applied to the proposed SIA procedure under the optimum conditions. The method was found to be linear, with a correlation coefficient of 0.9998 , in the range of $10-150 \mu \mathrm{g} / \mathrm{mL}$. The weighed regression of calibration is described in equation 2. "A" is the absorbance of the oxidized form of diclofenac. " $\mathrm{C}$ " is the concentration of diclofenac. Figure 3 shows a SIA-gram obtained by a one-shot run of four standard 


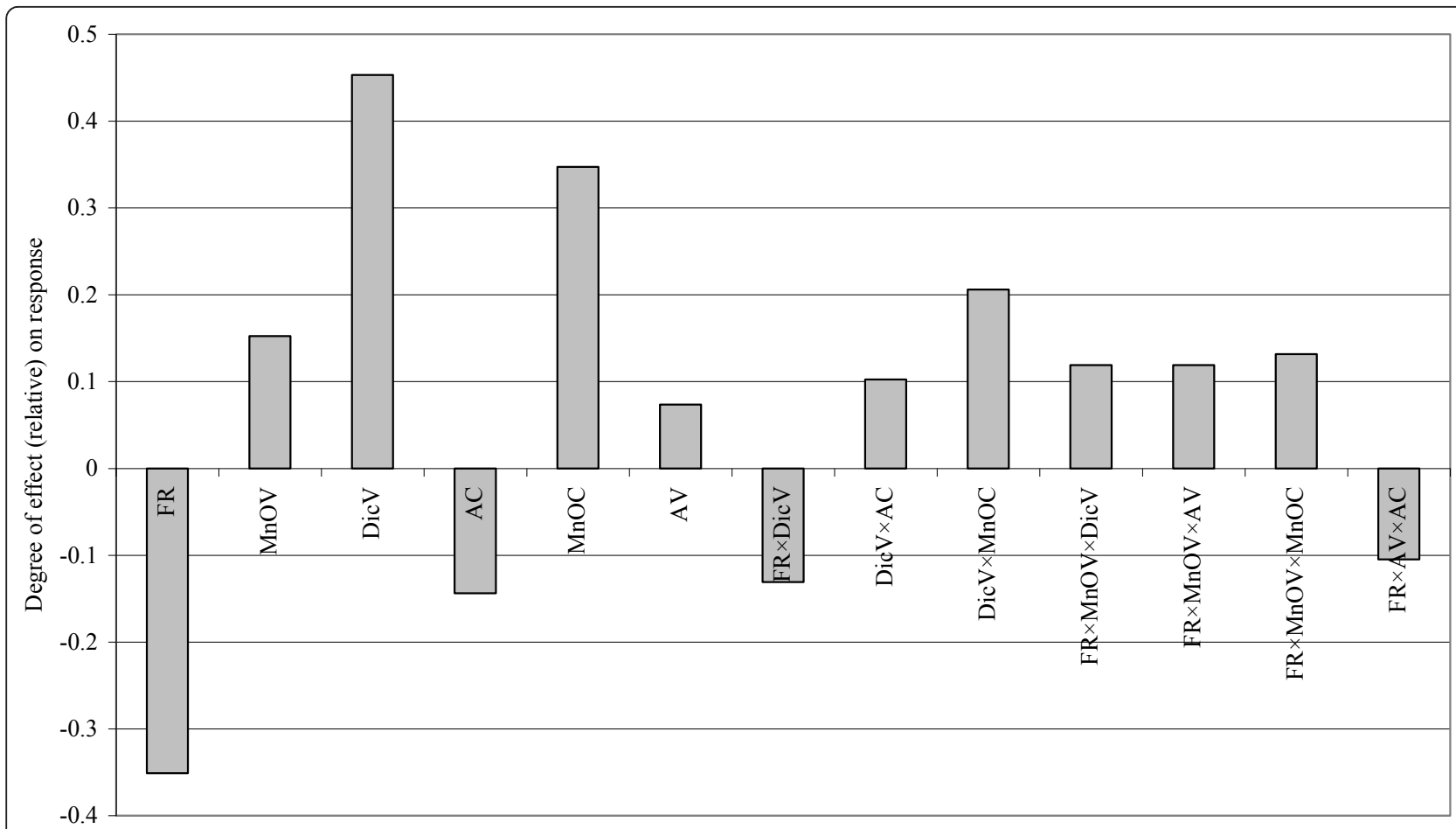

All main factors and most effective iteraction factors

Figure 2 Most effective main and interacting conditions on the response of the SIA method. FR: flow rate $(\mu \mathrm{L} / \mathrm{s})$, MnOV: permanganate volume $(\mu \mathrm{L})$, DicV: diclofenac volume $(\mu \mathrm{L}), \mathrm{AV}$ : acid volume $(\mu \mathrm{L}), \mathrm{AC}$ : acid concentration (mmol/L), MnOC: permanganate concentration (mmol/L).

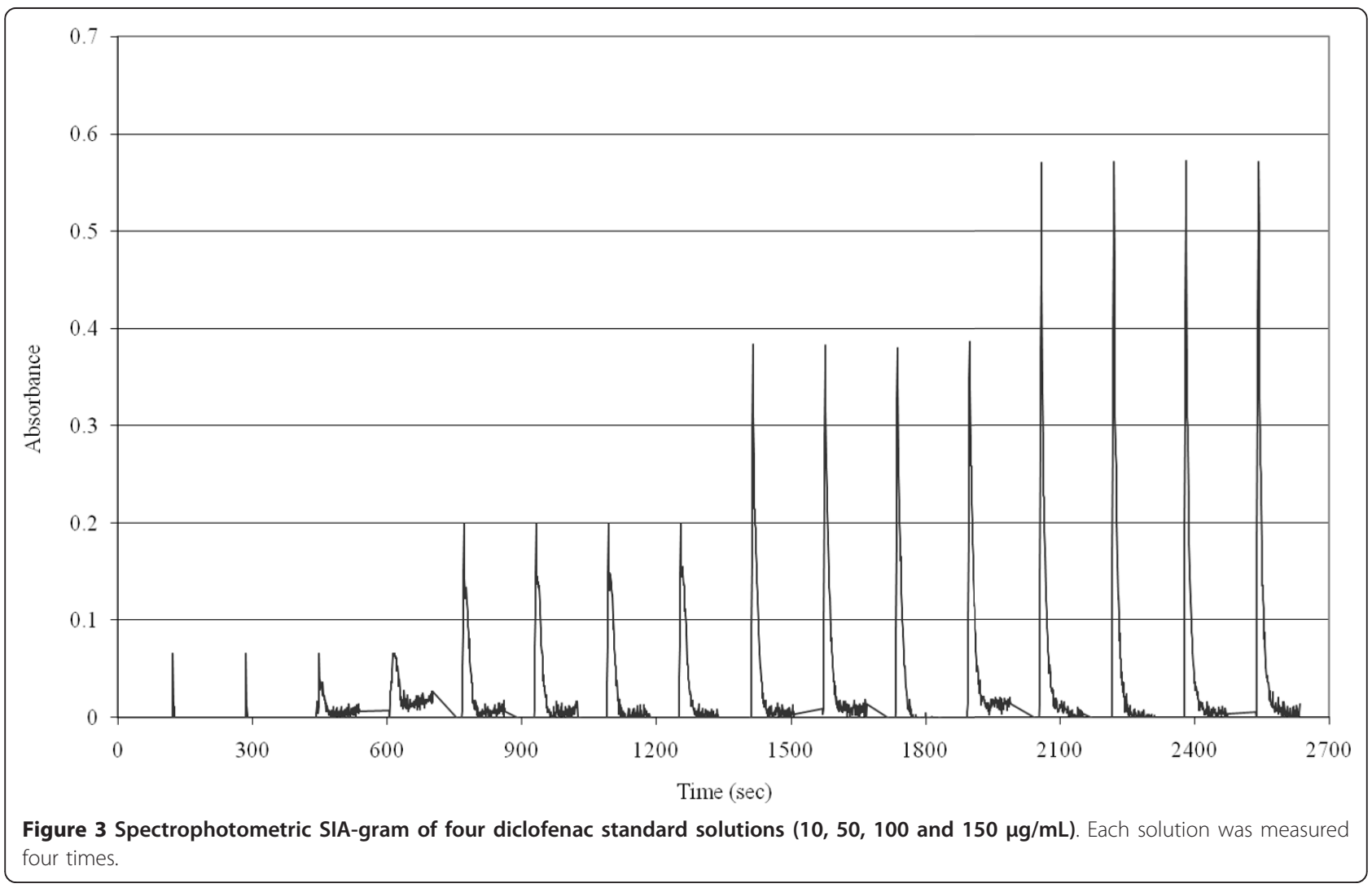


solutions $(10,50,100$ and $150 \mu \mathrm{g} / \mathrm{mL})$ of diclofenac; each standard solution was measured four times.

$$
\mathrm{A}=0.003 \mathrm{C}+0.024
$$

To examine the repeatability and the intermediate-precision, a standard solution of $50 \mu \mathrm{g} / \mathrm{mL}$ diclofenac was applied to the SIA method seven times in a day and five times over a week, respectively. The relative standard deviation (RSD) for repeatability study was $1.34 \%$ while the RSD for inter-mediate precision was $2.75 \%$. The automation of SIA rendered the proposed method precise.

The limits of detection (LOD) and quantification (LOQ) were also examined. LOD was obtained as the concentration of a solute resulting in a peak height three times the baseline noise level. LOQ was obtained as the concentration of solute resulting in a peak height ten times the baseline noise level. The LOD and LOQ were found to be 1.37 and $4.57 \mu \mathrm{g} / \mathrm{mL}$, respectively. Satisfactorily detectability of the SIA method was obtained by successful optimization.

\section{Method application}

The method was applied to bulk and pharmaceutical samples, namely tablets, injection and gel. Bulk, tablets and injection samples were also applied to the British Pharmacopoeia (BP) methods [47]. The BP recommends a classical potentiometric method for diclofenac assay in raw materials while a LC method is recommended for tablet and gel formulations [47]. For diclofenac assay in injection formulation, a previous validated HPLC method was applied [48]. Each sample was analyzed seven times. The recovery, RSD and $t$-test values were calculated. The obtained results are introduced in Table 2. The experimental $t$-test values were lower than those tabulated values, which prove the reliability of the current SIA method.

\section{Experimental}

\section{Instrumentation}

The assembly constructed for the current work included a SIA system, miniaturized fiber optic spectrometric devices and pumped-tubes (Figure 4).

\begin{tabular}{|c|c|c|c|c|}
\hline Trade name & Formulation & $\begin{array}{l}\text { Diclofenac } \\
\text { content }\end{array}$ & $\begin{array}{l}\text { Mean recovery } \pm \\
\operatorname{RSD}(\%)^{1}\end{array}$ & $t^{2}$ \\
\hline$\overline{\text { Samf }^{(\mathbb{R})}}$ & Bulk & - & $99.3 \pm 1.45$ & 2.14 \\
\hline OlfenTM-25 & Tablets & $25 \mathrm{mg}$ & $98.5 \pm 2.14$ & 2.06 \\
\hline OlfenTM-50 & Tablets & $50 \mathrm{mg}$ & $98.1 \pm 2.81$ & 2.11 \\
\hline Olfen ${ }^{\mathbb{B}}-75$ I.M. & Injection & $\begin{array}{l}75 \mathrm{mg} \\
\text { in } 2 \mathrm{ml}\end{array}$ & $99.1 \pm 2.26$ & 1.87 \\
\hline Voltaren ${ }^{\circledR}$ & Gel & $1 \%(w / w)$ & $103.4 \pm 3.49$ & 2.23 \\
\hline Diclogesic $^{\circledR}$ & Gel & $1 \%(\mathrm{w} / \mathrm{w})$ & $102.7 \pm 3.18$ & 2.70 \\
\hline
\end{tabular}

1: RSD: relative standard deviation for 7 replicates; 2 : t-test value.
The SIA system is a FIALab ${ }^{\circledR} 3500$ (Medina, WA USA). It is composed of a syringe pump (SP), multiposition valve (MPV), holding coil (HC), Z-flow cell (Z), pump tubing and personal computer (PC). The SP includes 24,000 increments with high-resolution stepper motor, which drives the piston at rates from 1.5 seconds to 10.0 min per stroke with $>99 \%$ accuracy at full stroke. The syringe has a volume of 2.5 $\mathrm{mL}$. The MPV is chemically inert and has eight ports with a standard pressure of $250 \mathrm{psi}$ (gas)/600 psi (liquid); zero dead volume. The $\mathrm{Z}$ is a $10 \mathrm{~mm}$ pathlength Plexiglass compatible with fiber optic connectors. Pump tubing was used to connect sequential injection analyzer devices and to make $\mathrm{HC}$ with a long of $200 \mathrm{~cm}$. Pumped tubes of " 0.03 inch" ID Teflon type was supplied from Upchurch Scientific, Inc. (Oak Harbor, WA, USA).

The optical manifold included a radiation source, spectrometer and fiber optic connectors. All optical devices were fabricated by Ocean Optics (Dunedin Florida, USA). The radiation source is an LS-1 Tungsten Halogen Lamb optimized for VIS-NIR (360 nm - $2 \mu$ m wavelength range). The detector is a USB2000 Spectrometer adapted to $200-1100 \mathrm{~nm}$ wavelength range. The fiber optic connectors are 200 micron Sub-Miniature version $A^{\circledR}$.

FIALab ${ }^{\circledR}$ for Windows version 5.0 supplied from FIAlab (Medina, WA, USA) was used for programming and controlling the whole assembly.

\section{Chemicals and reagents}

All chemicals and reagents, which were used in this study, were of analytical reagent grade. The quality of water was distilled deionized. Diclofenac sodium was supplied from Sigma (Taufkirchen, Germany). Potassium permanganate and sulphuric acid were supplied from Fluka (Buchs, Switzerland). Diclofenac sodium in the bulk form as well as inactive ingredients those possibly found in pharmaceutical formulations were a generous gift from Samf ${ }^{\circledR}$ Medicinal Factory (Khartoum North, Sudan). Inactive ingredients included sodium citrate, microcrystalline cellulose, magnesium stearate, maize starch, carnauba wax, povidone and talc.

\section{Pharmaceutical samples}

Olefn ${ }^{\mathrm{TM}}-25$ tablets (25 mg diclofenac sodium), Olefn $^{\text {TM }}-50$ tablets $\left(50 \mathrm{mg}\right.$ diclofenac sodium), Olefn ${ }^{\circledR}$ 75 I.M. (75 mg diclofenac sodium) ampoules, which were prepared by Mepha Ltd. Aesch-Basel, Switzerland, were examined in the current study. Voltaren ${ }^{\circledR}$ gel $(1 \%(\mathrm{w} / \mathrm{w})$ diclofenac sodium), which was prepared by Novartis, Aesch-Basel, Switzerland, was also examined. Diclogesic ${ }^{\circledR}$ gel $(1 \%(\mathrm{w} / \mathrm{w})$ diclofenac sodium) that was prepared by Dar Al-Dawa, Naur, Jordan was examined as well. 


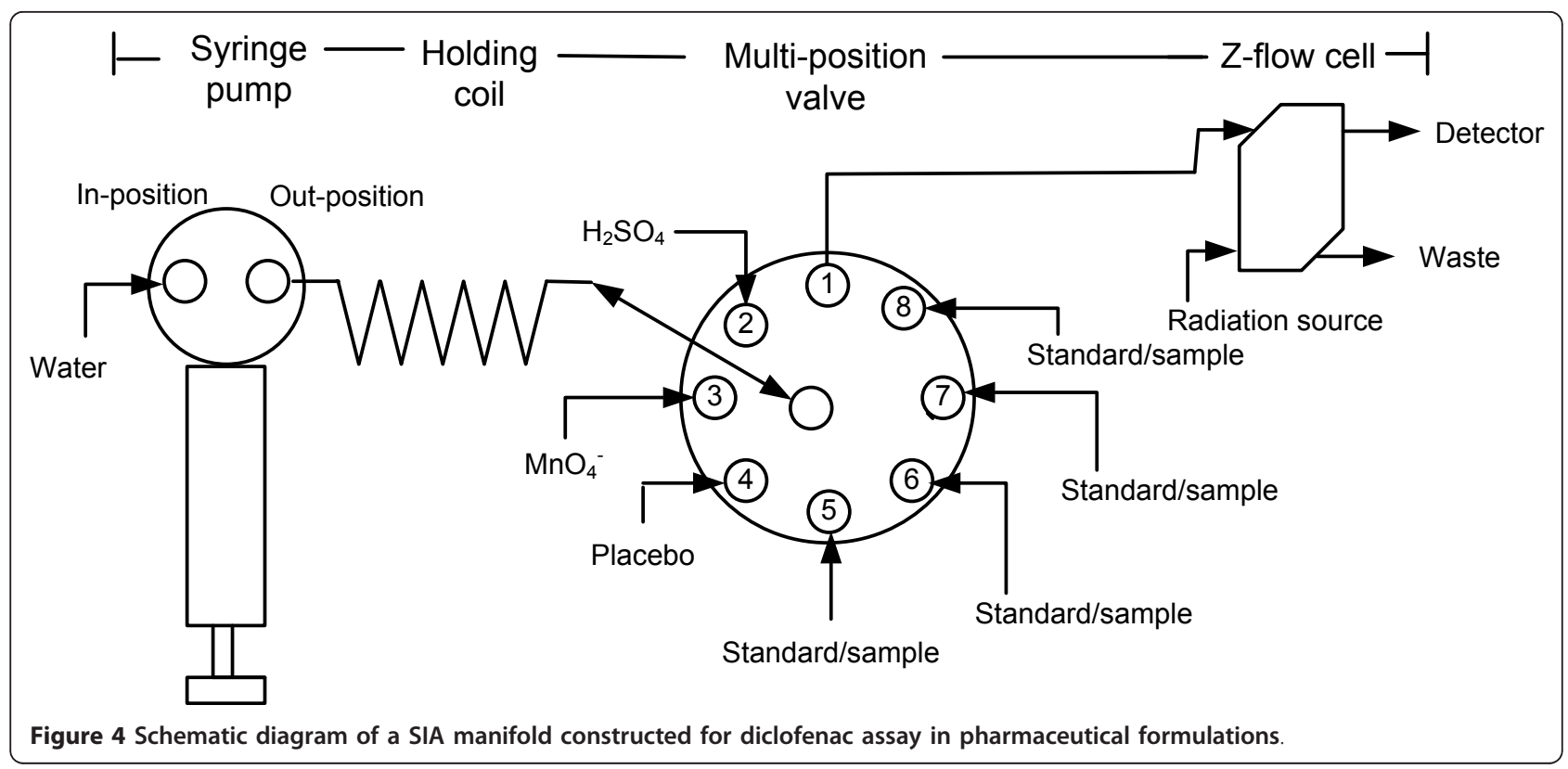

\section{Preparation of reagents and standard solutions}

A standard stock solution of $20 \mathrm{mmol} / \mathrm{L}$ potassium permanganate was prepared and standardized weekly in an appropriate way. An appropriate amount of diclofenac was dissolved in water to prepare $1000 \mu \mathrm{g} / \mathrm{mL}$ as stock standard solution. Working standard solutions of diclofenac, potassium permanganate and sulfuric were daily prepared by dilution.

\section{Preparation of pharmaceutical samples}

Twenty tablets were triturated and homogenized. An appropriate quantity, which is equivalent to $50 \mu \mathrm{g} / \mathrm{mL}$ diclofenac, was weighed and dissolved in $10 \mathrm{~mL}$ of water. Then, the obtained solution was heated in waterbath at $85^{\circ} \mathrm{C}$ for $5 \mathrm{~min}$ and centrifuged for $5 \mathrm{~min}$. The supernatant was filtered directly into a volumetric flask with an appropriate volume. The remaining material in the tube was treated two times again with hot water according to a previous procedure [47]. Finally, after cooling at room temperature, water was added to the solution to complete the volume of the volumetric flask.

For injection preparation, ten ampoules were discharged and mixed. An adequate volume was diluted to obtain $50 \mu \mathrm{g} / \mathrm{mL}$ diclofenac.

For gel preparation, five tubes were released. An accurately weighed portion of gel was treated as the tablet preparation procedure [47].

\section{Sequential injection analysis procedure}

As shown in Figure 4, a single-channel SIA manifold was constructed to perform on-line developing reaction and spectrophotometric measurement. The $\mathrm{Z}$ was attached to port-1 in the MPV. The radiation source and the spectrometer were connected with the $\mathrm{Z}$ by fiber optic connecters. Water, as a propelling solution, was linked with the in-position mode. Sulphuric acid, permanganate and placebo solutions were linked with port-2 to 4, respectively, in the MPV. Four standard/ sample solutions were attached to port-5 to -8 . As briefly described below, a rapid protocol controlling the proposed SIA procedure was programmed.

i. Following the practice of SIA, each solution was loaded into their relative tubes by aspiration using the SP. Then, excess volumes were dispensed to the waste.

ii. To propel solutions, the syringe was filled with $1500 \mu \mathrm{L}$ of water.

iii. For blank measurement, acid and permanganate solutions were sequentially aspirated into the $\mathrm{HC}$.

iv. The solutions were mixed using three times reverse-flow of $10 \mu \mathrm{L}$ volume at a flow rate of $10 \mu \mathrm{L} / \mathrm{s}$.

v. A placebo solution was injected into the $\mathrm{HC}$ and mixed with acidified permanganate as in step iv. vi. The mixture was dispensed through $\mathrm{Z}$ at the required flow rate. The peak height (PA) of absorbance was recorded.

vii. For standard/sample measurement, steps iv-vi were repeated with replacing a standard/sample solutions instead of a placebo solution.

\section{Conclusions}

The current work deals with the screening of conditions controlling spectrophotometric SIA and developing a 
new assay method for diclofenac. From the obtained results, the following conclusions can be made.

i. Full-factorial design is a powerful tool for the screening of conditions controlling SIA. It is also powerful for optimizing conditions at initial stage. However, other such chemometric approaches as fractional-factorial design, response surface and simplex could be more powerful for further optimization stages.

ii. In developing vis-spectrophotometric SIA methods those involve a developing reaction with two reagents (major and minor), it has been found that the main factors with their effect types, i.e. positive or negative, were ordered as follows: (+ sample volume $)>(-$ flow rate $) \approx(+$ major reagent concentration $)>>(+$ major reagent volume $) \approx(-$ minor reagent concentration $)>>(+$ minor reagent volume $)$. iii. It has been also found that there was a significant interaction effect between sample volume and major reagent concentration.

iv. Generally, in SIA that involves a developing reaction, it is recommended to utilize a chemometric tool for optimizing effective conditions (i.e. sample volume, flow rate and major reagent concentration) while less effective conditions could be fixed at suitable levels.

\section{Acknowledgements}

The author expresses his gratitude to the financial support from King Abdulaziz City for Science and Technology, Saudi Arabia, award \# MT-3-6. Thanks also are due to the Department of Chemistry, College of Science, King Faisal University for allowing the author to conduct this work.

\section{Authors' information}

Dr. Abubakr M. Idris received his BSc (1994), MSc (1999) and PhD (2005) from the University of Khartoum, Khartoum, Sudan. He is an MRSC and active member of the American Chemical Society. Dr. Idris is currently an Assistant Professor at the Department of Chemistry, College of Science, King Faisal University, Hofuf, Saudi Arabia. Idris has co-authored more than forty papers in international refereed journals and conferences. His research focuses on developing microfluidic analytical technologies and their assay methodologies. He has some publications on environmental analytical issues as well.

\section{Competing interests}

The author declares that they have no competing interests.

Received: 23 October 2010 Accepted: 20 February 2011 Published: 20 February 2011

\section{References}

1. Ruzicka J, Marshall GD: Sequential injection: A new concept for chemical sensors, process analysis and laboratory assays. Analytica Chimica Acta 1990, 237(2):329-343

2. Idris AM: An overview of the generations and recent versions of flow injection techniques. Critical Reviews in Analytical Chemistry 2010, 40(3):150-158.

3. Idris AM: Flow injection, overlooked techniques in forensic analysis. Critical Reviews in Analytical Chemistry 2010, 40(4):218-225.

4. Morgan ED: Chemometrics: Experimental Design. ACOL, John Wiley and Sons, London, UK; 1991.
5. Douglas C: Montgomery, Design and analysis of experiments. John Wiley and Sons, Inc, New York; Fourth 1997.

6. Miller JN, Miller JC: Statistics and Chemometrics for Analytical Chemistry. Pearson Prentice Hall, Harlow, UK, 52004.

7. Solich P, Sklenarova H, Polaek M, Karlícek R: Application of flow injection technique in pharmaceutical analysis. Part II.: Spectrophotometric and chemiluminescence detection. Journal of Flow Injection Analysis 2001, 18(2):118-125.

8. Lenehan CE, Barnett NW, Lewis SW: Sequential injection analysis. Analyst 2002, 127(8):997-1020.

9. Pimenta AM, Montenegro MCBSM, Araujo AN, Calatayud JM: Application of sequential injection analysis to pharmaceutical analysis. Journal of Pharmaceutical and Biomedical Analysis 2006, 40(1):16-34.

10. Tzanavaras PD, Themelis DG: Review of recent applications of flow injection spectrophotometry to pharmaceutical analysis. Analytica Chimica Acta 2007, 588(1):1-19.

11. Gomez V, Callao MP: Use of multivariate curve resolution for determination of chromium in tanning samples using sequential injection analysis. Analytical and Bioanalytical Chemistry 2005, 382(2):328-334.

12. Gomez V, Pasamontes A, Callao MaP: Factorial design for optimising chromium determination in tanning wastewater. Microchemical Journal 2006, 83(2):98-104

13. Pasamontes A, Callao MP: Optimization by means of responses surface of an analytical sequence using a sequential injection system. Talanta 2006, 68(5):1617-1622

14. Callao MP: Fractional factorial design and simplex algorithm for optimizing sequential injection analysis (SIA) and second order calibration. Chemometrics and Intelligent Laboratory Systems 2006, 83(2):127-132

15. Pasamontes A, Callao MP: Sequential injection analysis linked to multivariate curve resolution with alternating least squares. Trends in Analytical Chemistry 2006, 25(1):77-85.

16. Cladera A, Gomez E, Estela JM, Cerda V: Effect of variables influencing $S(1 /$ 4) in sequential injection analysis. Extrapolability of $S(1 / 4)$ based results between SIA designs. Talanta 1996, 43(10):1667-1674.

17. Sultan SM, Hassan YAM, Ibrahim KEE: Sequential injection technique for automated titration: Spectrophotometric assay of vitamin $C$ in pharmaceutical products using cerium(IV) in sulfuric acid. Analyst 1999, 124(6):917-921.

18. Sultan SM, Hassan YAM, Ibrahim KEE: Sequential injection spectrophotometric assay of bromazepam complexed with iron(II) in hydrochloric acid with chemometric optimization. Talanta 1999, 50(4):841-849.

19. Sultan SM, Idris AM, Ibrahim KE: Sequential injection spectrophotometric method for the assay of paracetamol in drug formulations. Journal of Flow Injection Analysis 2004, 21(1):19-24.

20. Moreno CL, Rudner PC, Garcia JMC, Pavon JMC: Development of a sequential injection analysis device for the determination of total polyphenol index in wine. Microchimica Acta 2004, 148(1-2):93-98.

21. Idris AM, Assubaie FN, Sultan SM: Chemometric optimization of a SIA promethazine hydrochloride assay method. Microchemical Journal 2006, 83(1):7-13.

22. De Oliveira FS, Korn M: Spectrophotometric determination of sulphate in automotive fuel ethanol by sequential injection analysis using dimethylsulphonazo(III) reaction. Talanta 2006, 68(3):992-899.

23. Idris AM: On-line coupling of solid-phase extraction, derivatization reaction and spectrophotometry by sequential injection analysis: Application to trifluoperazine assay in human urine. Journal of Pharmacological and Toxicological Methods 2007, 56(3):330-335.

24. Idris AM, Assubaie FN, Sultan SM: Experimental design optimization of a sequential injection method for promazine assay in bulk and pharmaceutical formulations. Journal of Automated Methods and Management in Chemistry 2007, 32470.

25. Allan CV, dS Jorge CM: Applying sequential injection analysis (SIA) and response surface methodology for optimization of Fenton-based processes. Talanta 2009, 77(3):1081-1086.

26. Idris AM: Factorial design and response surface optimization of spectrophotometric sequential injection analysis of olanzapine formulations. Journal of Analytical Chemistry 2010, 65(1):36-42. 
27. Tubino M, De Souza RL: Gravimetric method for the determination of diclofenac in pharmaceutical preparations. Journal of AOAC International 2005, 88(6):1684-1687.

28. Mitic S, Miletic G, Pavlovic A, Tosic S, Pecev E: Determination of diclofenac sodium in commercial pharmaceutical formulations and human control serum using a kinetic-spectrophotometric method. Chemical and Pharmaceutical Bulletin 2007, 55(10):1423-1426.

29. Castellano PM, Vignaduzzo SE, Maggio RM, Kaufman TS: Application of a chemometric method for simultaneous determination of acetaminophen and diclofenac in content-uniformity and drug-dissolution studies. Analytical and Bioanalytical Chemistry 2005, 382(7):1711-1714.

30. Castillo MA, Bruzzone L: Indirect fluorometric determination of diclofenac sodium. Analytical Sciences 2006, 22(3):431-433.

31. Mazurek S, Szostak R: Quantitative determination of diclofenac sodium and aminophylline in injection solutions by FT-Raman spectroscopy. Journal of Pharmaceutical and Biomedical Analysis 2006, 40(5):1235-1242.

32. Mazurek S, Szostak R: Quantitative determination of diclofenac sodium in solid dosage forms by FT-Raman spectroscopy. Journal of Pharmaceutical and Biomedical Analysis 2008, 48(3):814-821.

33. Tubino M, De Souza RL: Determination of diclofenac in pharmaceutical preparations by diffuse reflectance photometry. Talanta 2006, 68(3):776-780.

34. Santini AO, Pezza HR, Pezza L: Determination of diclofenac in pharmaceutical preparations using a potentiometric sensor immobilized in a graphite matrix. Talanta 2006, 68(3):636-642.

35. Kormosh Z, Hunka I, Bazel Y: Potentiometric determination of diclofenac in pharmaceutical formulation by membrane electrode based on ion associate with base dye. Chinese Chemistry Letters 2007, 18(9):1103-1106.

36. Rodriguez JE, Barrado E, Castrillejo Y, Santos JR, Lima JLFC: Validation of a tubular bismuth film amperometric detector. Determination of diclofenac sodium by multisyringe flow injection analysis. Journal of Pharmaceutical and Biomedical Analysis 2007, 45(1):47-53.

37. Asfak V, Mrinalini D, Leena B, Rahul G: Simultaneous determination of diclofenac sodium and rabeprazole sodium in bulk and pharmaceutical dosage form by LC. Chromatographia 2007, 66(11-12):941-493.

38. Bhushan R, Gupta D, Mukherjee A: Liquid chromatographic analysis of certain commercial formulations for non-opioid analgesics. Biomedical Chromatography 2007, 21(12):1284-1290.

39. Shaikh KA, Devkhile AB: Simultaneous determination of aceclofenac, paracetamol, and chlorzoxazone by RP-HPLC in pharmaceutical dosage form. Journal of Chromatographic Science 2008, 46(7):649-652.

40. Bhinge JR, Kumar RV, Sinha VRA: A simple and sensitive stabilityindicating RP-HPLC assay method for the determination of aceclofenac. Journal of Chromatographic Science 2008, 46(5):440-444.

41. Multari G, Incarnato G, Gagliardi L: High-performance liquid chromatography analysis of anti-inflammatory pharmaceuticals with ultraviolet and electrospray-mass spectrometry detection in suspected counterfeit homeopathic medicinal products. Journal of Pharmaceutical and Biomedical Analysis 2007, 43(4):1221-1227.

42. Hopkala H, Pomykalski A: TLC analysis of non-steroidal anti-inflammatory drugs and videodensitometric determination of fenbufen in tablets. Journal of Planar Chromatography - Modern TLC 2004, 17(5):383-387.

43. Dighe W, Sane RT, Menon SN, Tambe HN, Pillai S, Gokarn VN: Simultaneous determination of diclofenac sodium and paracetamol in a pharmaceutical preparation and in bulk drug powder by highperformance thin-layer chromatography. Journal of Planar Chromatography - Modern TLC 2006, 19(112):443-448.

44. Dowling G, Gallo P, Fabbrocino S, Serpe L, Regan L: Determination of ibuprofen, ketoprofen, diclofenac and phenylbutazone in bovine milk by gas chromatography-tandem mass spectrometry. Food Additives and Contaminants - Part A Chemistry, Analysis, Control, Exposure and Risk Assessment 2008, 25(12):1497-1508.

45. Sultan SM, Idris AM, Ibrahim KEE: Sequential injection kinetic method for the assay of aspirin in drug formulations. Journal of Flow Injection Analysis 2005, 22(2):118-122.

46. Idris AM, Sultan SM, Ibrahim KEE, Assubaei FN: Sequential injection spectrophotometric kinetic method for the determination of paracetamol in dosage forms. Journal of Flow Injection Analysis 2005, 22(2):123-128.

47. British Pharmacopoeia Ver. 6.0. London, United Kingdom: British Pharmacopoeia Commission Secretariat; 2002.
48. Gonzalez L, Yuln G, Volonte GM: Determination of cyanocobalamin, betamethasone, and diclofenac sodium in pharmaceutical formulations, by high performance liquid chromatography. Journal of Pharmaceutical and Biomedical Analysis 1999, 20(3):487-492.

doi:10.1186/1752-153X-5-9

Cite this article as: Idris: Screening of conditions controlling spectrophotometric sequential injection analysis. Chemistry Central Journal 2011 5:9.

Publish with ChemistryCentral and every
scientist can read your work free of charge
"Open access provides opportunities to our
colleagues in other parts of the globe, by allowing
anyone to view the content free of charge."
W. Jeffery Hurst, The Hershey Company.
- available free of charge to the entire scientific community
- peer reviewed and published immediately upon acceptance
- yours - you keep the copyright
submit your manuscript here:
http://www.chemistrycentral.com/manuscript/

